\title{
Influência da distância da borda e do adensamento foliar sobre a abundância de plantas pioneiras em um fragmento de floresta tropical submontana na Estação Ecológica de Wenceslau Guimarães (Bahia, Brasil)
}

Dary Moreira Gonçalves Rigueira ${ }^{1,2,3}$, Adam Luís Minho Molinari ${ }^{1}$, Dante Luís Silva Mariano ${ }^{1}$, Rodrigo Maman Reis ${ }^{1}$, Adriana Brizon Portugal ${ }^{1}$, Neildes de Souza Santana ${ }^{1}$ e Rafael Alves dos Santos ${ }^{1}$

Recebido em 18/05/2011. Aceito em 6/02/2012

\begin{abstract}
RESUMO
(Influência da distância da borda e do adensamento foliar sobre a abundância de plantas pioneiras em um fragmento de floresta tropical submontana na Estação Ecológica de Wenceslau Guimarães (Bahia, Brasil)). Efeitos de borda são modificações nos parâmetros físicos, químicos e biológicos observados na área de contato da margem da floresta com a matriz circundante, sendo a disponibilidade de luz um fator crucial para o seu desencadeamento. O objetivo do estudo foi avaliar a influência da disponibilidade de luz sobre a abundância de espécies pioneiras no interior de fragmentos de uma floresta tropical submontana, no município de Wenceslau Guimarães, Bahia. Os resultados mostram uma redução da abundância de espécies pioneiras com o aumento da distância em relação à borda, sendo explicada pelo aumento do adensamento foliar, e conseqüente diminuição da disponibilidade de luz a partir de 30 metros de distância da borda. O entendimento deste padrão se mostra satisfatório ao explicar a distribuição de plantas pioneiras em paisagens fragmentadas, sendo útil no desenvolvimento de estratégias de gestão visando o manejo adequado da paisagem.
\end{abstract}

Palavras-chave: efeito de borda, disponibilidade de luz, estrato florestal, fragmentação

\begin{abstract}
(Influence of edge distance and leaf density on the abundance of pioneer plants in a patch of tropical submontane forest in the Ecological Station of Wenceslau Guimarães (Bahia, Brazil)). Edge effects are changes in physical, chemical and biological parameters observed in the area of contact of the forest edge with the surrounding matrix. Light availability is the crucial trigger factor. The aim of this study was to assess the influence of light availability on abundance of pioneer plant species in a fragment of a tropical submontane forest, in the municipality of Wenceslau Guimarães, Bahia, Brazil. The results showed reduced abundance of pioneer species as edge distance increased. This can be explained by increased leaf density, and consequently reduction of light availability as of 30 meters from the edge in the various forest strata. This pattern explains the distribution of pioneer plants in fragmented landscapes, and is useful in the development of management strategies such as proper management of the landscape.
\end{abstract}

Key words: edge effect, light availability, forest strata, fragmentation

\section{Introdução}

Os processos de perda e fragmentação florestal são considerados como um dos principais promotores de distúrbios ambientais em ecossistemas terrestres. Esses processos, que podem ocorrer naturalmente, derivam principalmente da ação antrópica, alterando as dinâmicas populacionais e estruturas das comunidades locais, além de modificarem os fatores abióticos nos fragmentos florestais (Gehlhsausen et al. 2000; Franklin et al. 2002; Fahrig 2003; Tabarelli et al. 2006; Watling \& Orrock 2010). Uma alteração marcante em paisagens fragmentadas é o incremento de bordas, formando uma transição geralmente abrupta entre a margem da floresta e a matriz ao seu redor (Tabarelli et al. 1999). A interação mútua entre esses dois ambientes adjacentes resulta nos efeitos de borda, tanto para os hábitats florestais remanescentes quanto às matrizes desmatadas (Murcia 1995; Tabarelli 2004).

\footnotetext{
1 Universidade Federal da Bahia, Programa de Pós-Graduação em Ecologia e Biomonitoramento, Salvador, BA, Brasil

2 Universidade Federal da Bahia, Instituto de Biologia, Departamento de Botânica, Laboratório Flora, Salvador, BA, Brasil

3 Autor para correspondência: daryrigueira@yahoo.com.br
} 
É amplo o espectro de alterações e efeitos oriundos das bordas em fragmentos florestais sobre as comunidades vegetais associadas (Murcia 1995; Ries et al. 2004; Harper et al. 2005). Dentre esses efeitos, a disponibilidade de luz é considerada como um fator crucial, influenciando diretamente a temperatura e a umidade (Bazzaz 1979; Murcia 1995; Dignan \& Bren 2003; Figueiró \& Coelho Netto 2007). Assim, diferenças na disponibilidade de luz entre a borda e o interior de fragmentos florestais têm importantes implicações para a distribuição das espécies de plantas (Matlack 1993; Gehlhausen et al. 2000; Gonzales et al. 2010; Watling \& Orrock 2010). A vegetação de borda, com menor porte e menor adensamento foliar possibilita uma maior penetração da luz, tanto vertical quanto lateral, estimulando a germinação e crescimento de espécies heliófitas, sendo estas geralmente pioneiras, que apresentam taxas fotossintéticas mais elevadas do que as espécies secundárias e tolerantes à sombra, tornando-as mais competitivas e abundantes nestes ambientes de borda (Bazzaz 1979; Brothers \& Springarn 1992; Jose et al. 1996; Laurance 2000, Valladares et al. 2000; Nascimento et al. 2006; Laurance \& Curran 2008).

Os efeitos proporcionados pela formação da borda, como o aumento da disponibilidade de luz, tendem a diminuir de intensidade à medida que se afasta da borda em direção ao interior do fragmento e, conseqüentemente, há uma redução na exposição das plantas a esses efeitos (Murcia 1995; Fox et al. 1997; Ries et al. 2004; Harper et al. 2005). Estudos realizados com plantas pioneiras ou invasoras em relação à disponibilidade de luz indicam que, freqüentemente, a partir de $50 \mathrm{~m}$ da borda, esses efeitos são praticamente anulados, assemelhando-se ao ambiente do interior do fragmento (Murcia 1995; Ries et al. 2004). Todavia, valores discrepantes têm sido encontrados em outros trabalhos realizados em florestas tropicais úmidas, variando de apenas 4-12 m (Fox et al. 1997), até $500 \mathrm{~m}$ de distância da borda (Laurance 1991). Estes resultados distintos revelam a amplitude de variação das respostas em diferentes áreas florestadas, frente aos efeitos de borda, favorecendo assim a realização de novos estudos empíricos que avaliem tal questão.

Para uma melhor compreensão sobre possíveis efeitos de borda em paisagens fragmentadas, a avaliação integrada entre fatores bióticos e abióticos é imprescindível (Gehlhausen et al. 2000; Watling \& Orrock 2010). Desta forma, buscamos avaliar indiretamente a influência da disponibilidade de luz, através da medida de adensamento foliar em estratos florestais, e da distância entre borda-interior sobre a abundância de plantas pioneiras em fragmentos de uma floresta tropical úmida submontana.

Nossa expectativa é de que a abundância de plantas pioneiras diminua à medida que se aumenta a distância da borda, limitada pela maior densidade de folhas e, consequente, menor disponibilidade de luz no interior dos fragmentos.

\section{Materiais e métodos}

\section{Área de Estudo}

O estudo foi desenvolvido na Estação Ecológica Estadual de Wenceslau Guimarães (EEEWG), uma Unidade de Conservação (UC) na categoria de Proteção Integral (SNUC Lei No 9.985/2000 e Decreto No 4.340/2002), criada pelo Decreto Estadual No 6.228/1997 e ampliada pelo Decreto Estadual No 7.791/2000, localizada no município de Wenceslau Guimarães, região do Baixo Sul da Bahia, totalizando uma área de 2.418 hectares, estando a sede da EEEWG localizada nas coordenadas geográficas $13^{\circ} 35^{\prime} 43^{\prime \prime} \mathrm{S}$ e 39³'10"W (Bahia 2010).

Esta UC está inserida no Bioma Mata Atlântica, tendo como tipo de vegetação a Floresta Ombrófila Densa de formação submontana, disposta em fragmentos florestais não contínuos. Sua geomorfologia é marcada por vales, quase sempre profundos e retilíneos que variam de largura entre $200 \mathrm{a} 500 \mathrm{~m}$. Os valores de declividade predominante variam entre $15^{\circ}$ a $50^{\circ}$, com cotas de altitude que variam entre 550 a 1000 metros. A predominância do tipo de solo é do Latossolo Vermelho-Amarelo Álico, podendo também ser encontrado Argissolo e Neossolo (Bahia 2010).

O clima da região é caracterizado como úmido à subúmido, apresentando transição entre a faixa de clima úmido ao leste e a faixa de clima subúmido ao oeste das Serras Marginais. A temperatura média anual varia entre $22^{\circ} \mathrm{e}$ $25,5^{\circ} \mathrm{C}$ e os totais pluviométricos encontram-se na faixa entre 800 a $1.500 \mathrm{~mm}$ anuais (Bahia 2010).

\section{Seleção das Plantas Pioneiras}

As plantas selecionadas para o estudo foram: capianga, Vismia guianensis (Aubl.) Choisy - Hypericaceae; mundururu, Miconia dodecandra (Desr.) Cobn. - Melastomataceae; embaúbas, Cecropia spp. - Urticaceae. Para os espécimes de embaúbas, foram quantificadas todas as espécies pertencentes ao gênero Cecropia.

Essas espécies vegetais dos três gêneros citados são classificadas como heliófitas e pioneiras, necessitando de luz abundante para seu desenvolvimento (Brasil 1994; Lorenzi 2000; Souza \& Lorenzi 2005), além de serem facilmente identificadas em campo.

\section{Seleção do Fragmento}

Foi escolhido um fragmento com área de 148 hectares e extensão de 1,88 km. Este apresentava bordas nitidamente marcadas, com visível divisão espacial entre fragmento florestal e matriz desmatada. Consideramos como matriz as pastagens abandonadas, estrada de barro ou áreas agricultáveis de baixo porte.

\section{Medida da Distância Borda-Interior}

Foram estabelecidas 21 transecções com $100 \mathrm{~m}$ de comprimento cada, perpendiculares à borda e com $5 \mathrm{~m}$ de largura. As transecções foram dispostas aleatoriamente em 
diferentes regiões do fragmento e distantes no mínimo a 100 $\mathrm{m}$ da transecção mais próxima. Em seguida as transecções foram divididas em 10 partes iguais de $10 \mathrm{~m}$ cada, sendo quantificada a abundância das espécies pioneiras supracitadas em cada porção de $10 \mathrm{~m}$ da transecção.

\section{Medida de Adensamento Foliar nos Estratos Florestais}

A medida de adensamento foliar nos estratos florestais foi obtida a partir da estimativa visual, por um único observador, através de um cilindro imaginário de $20 \mathrm{~cm}$ de diâmetro, colocado verticalmente do solo até a porção superior do dossel, sendo quantificada a porcentagem de preenchimento por folhas no cilindro em quatro estratos de diferentes alturas, a saber: estrato 1 , menor do que $5 \mathrm{~m}$; estrato 2, entre $5-10 \mathrm{~m}$; estrato 3, entre $10-20 \mathrm{~m}$; e estrato 4 , maior do que $20 \mathrm{~m}$. A altura do cilindro imaginário foi estimada com uso de uma trena digital. A cada $10 \mathrm{~m}$ da transecção eram feitas duas estimativas de adensamento foliar, uma em torno de $2,5 \mathrm{~m} \mathrm{e}$ a outra de 7,5 $\mathrm{m}$ do início de cada faixa de $10 \mathrm{~m}$ (evitando as clareiras), e a média desses valores foi considerada como o valor da distância da borda em questão. Estes valores foram utilizados como uma medida indireta da disponibilidade de luz no fragmento, ou seja, quanto maior for o valor de densidade foliar, maior será a interceptação da luz pelas folhas e menor será a disponibilidade de luz na área avaliada (Sanqueta 1995; Mourelle et al. 2001). Diferentemente de uma medida direta de luminosidade captada por aparelhos (p. ex. luxímetro), que poderá ser influenciado pelas condições do tempo (dia nublado e ensolarado) além do horário da medição (o sol do meio dia é mais intenso do que o do início da manhã), o adensamento foliar nos possibilita inferir sobre a disponibilidade de luz numa perspectiva dinâmica, através da competição foliar por luz.

\section{Análises Estatísticas}

Foram realizadas análises descritivas e multivariadas assumindo um $a=0,05$. Todas as análises estatísticas fo-

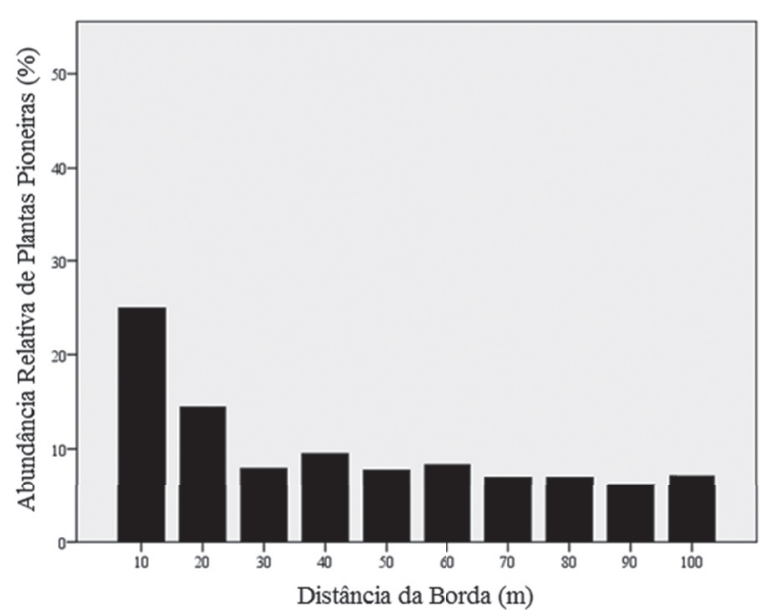

Figura 1. Abundância relativa de plantas pioneiras a cada 10 metros de distância da borda dos fragmentos. ram testadas quanto à tendência e normalidade dos dados, de acordo com os pressupostos existentes (Sheskin 2000, Sprent 2001). Todos os testes e análises foram executados no programa SPSS Statistics v.17 (SPSS 2010).

\section{Resultados}

Foram contabilizados ao todo 505 indivíduos heliófitos nas 21 transecções amostrados. Destes indivíduos, 39,41\% estavam presentes nos primeiros $20 \mathrm{~m}$ da transecção (Fig. 1). A média da abundância relativa das espécies pioneiras diminui significativamente (análise de regressão não linear em potência: $\left.\mathrm{p}=0,000 ; \mathrm{R}^{2}=0,866\right)$ de 6 indivíduos nos primeiros $10 \mathrm{~m}$ para 1,71 indivíduos nos últimos $100 \mathrm{~m}$ da borda (Fig. 2).

$\mathrm{O}$ adensamento foliar médio teve maiores médias e variâncias nos estratos 1 (até $5 \mathrm{~m})$ e $2(5-10 \mathrm{~m})$ em relação aos 3 (10-20 m) e 4 (maior que 20 m) (Tab. 1; Fig. 3; Fig. 4). O adensamento foliar médio também tendeu a se correlacionar positivamente ao longo da transecção de $100 \mathrm{~m}$, entretanto somente os estratos 2 e 4 demonstraram serem significativos (Tab. 1; Fig. 4). A independência e ordem natural desses estratos também se mostraram ser significativos (teste de Jonckheere-Terpstra: $\mathrm{p}=0,000)$.

Considerando o adensamento foliar médio total nos fragmentos florestais, os resultados mostraram um aumento significativo com a distância (análise de regressão linear: $\mathrm{p}<0,002 ; \mathrm{R}^{2}=0,73$ ), onde se tornou mais acentuado a partir dos $30 \mathrm{~m}$ de distância da borda (Fig. 5).

\section{Discussão}

A abundância relativa de plantas pioneiras ao longo do gradiente borda-interior do fragmento respondeu como o esperado, havendo uma redução mais acentuada destas espécies a partir de 30 metros da borda. Estes valores estão acima dos apresentados por Fox e colaboradores

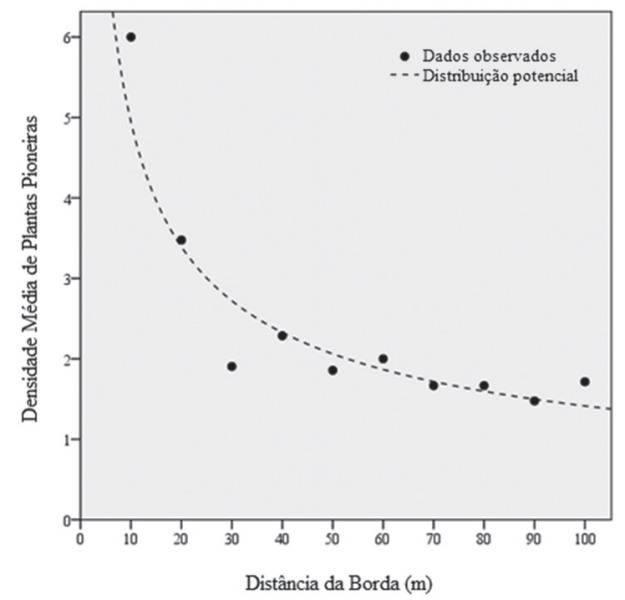

Figura 2. Regressão não linear de potência entre a densidade média de plantas pioneiras e a distância da borda dos fragmentos. 
Tabela 1. Resumo das análises descritivas e do teste de correlações Spearman’s rho para o adensamento foliar médio dos fragmentos.

\begin{tabular}{|c|c|c|c|c|c|}
\hline \multirow{2}{*}{ Análises } & \multicolumn{5}{|c|}{ Resultados } \\
\hline & Estrato 1 & Estrato 2 & Estrato 3 & Estrato 4 & Total \\
\hline Média & 49,25 & 62,14 & 24,95 & 5,21 & 141,55 \\
\hline Mediana & 48,09 & 63,75 & 25,63 & 5,16 & 145,42 \\
\hline Desvio padrão & 5,46 & 9,19 & 5,94 & 2,34 & 14,82 \\
\hline Variância & 29,79 & 84,49 & 35,26 & 5,49 & 219,78 \\
\hline \multicolumn{6}{|c|}{ Teste Spearman's rho (Estratos x Distância) } \\
\hline Coeficiente de correlação & 0,115 & 0,782 & 0,588 & 0,891 & \\
\hline Significância (2-tailed) & 0,751 & $0,008^{*}$ & 0,074 & $0,001^{*}$ & \\
\hline
\end{tabular}

*Significante ao $\alpha=0,05$

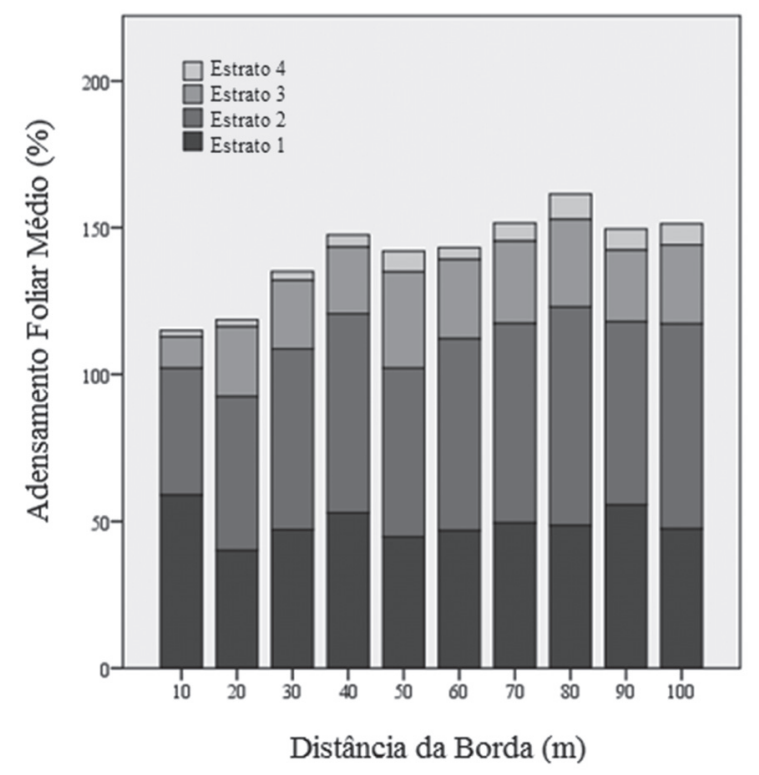

Figura 3. Adensamento foliar médio dos estratos florestais ao longo da distância da borda dos fragmentos.

(1997), de 4-12 m de penetração dos efeitos de borda dentro do fragmento florestal, numa floresta tropical úmida, e abaixo dos $40 \mathrm{~m}$ proposto por Gehlhsausen e colaboradores (2000), para florestas temperadas, e dos $500 \mathrm{~m}$ indicado por Laurence (1991), para uma floresta tropical úmida. Num trabalho de revisão, Murcia (1995) indica que na maioria das pesquisas sobre efeitos de borda, tanto para florestas temperadas quanto tropicais, desaparecem após 50 metros da borda. Contudo, a presença das plantas se manteve constante, porém baixa, ao longo de toda transecção, apresentando valores de densidade média, nos $70 \mathrm{~m}$ finais da transecção, entre 1 e 2 indivíduos apenas . O adensamento foliar, com conseqüente redução da disponibilidade de luz nos fragmentos florestais, tendeu a aumentar ao longo do gradiente borda-interior do fragmento, sendo demonstrado também em outros estudos realizados (Harper et al. 2005). Para ambientes florestais, a medida direta da quantidade de folhas e o número de ramos das árvores são as variáveis bióticas que mais explicam a diminuição da disponibilidade de luz (Mourelle et al. 2001), possibilitando estudos de estratificação florestal e um melhor entendimento das estratégias de regeneração natural, crescimento e sobrevivência de populações vegetais (Sanqueta 1995). Desta forma, o aumento da densidade foliar uma medida indireta da luminosidade no fragmento florestal, pode ser considerado como um dos principais fatores promotores de alterações em comunidades florestais em paisagens fragmentadas, podendo estar relacionado com a abundância de espécies pioneiras e heliófitas, encontradas na porção mais periférica do fragmento estudado (Bazzaz 1979; Murcia 1995; Williams-Linera et al. 1998).

Considerando o adensamento foliar médio e a abundância relativa de plantas heliófitas (Fig. 2) nas análises de regressão em potência, foi possível inferir que aos 30 metros há uma redução mais acentuada na abundância de plantas pioneiras devido ao aumento no adensamento foliar e conseqüente redução de luminosidade. Este intervalo encontrado está próximo do observado por Kapos (1989), ao qual apresenta níveis elevados da disponibilidade de luz até aos $40 \mathrm{~m}$ num estudo de fragmentos recém formados na Amazônia. Para bordas com um tempo maior de formação, a maior disponibilidade de luz fica restrita aos 10 metros inicias (Macdougall \& Kellman 1992; Matlack 1993), sugerindo que as bordas presentes na área do nosso estudo, com maior disponibilidade de luz até os 20 metros inicias, poderiam ter uma idade intermediária, pois apresenta um valor de distância abaixo dos 40 metros das bordas recém formadas e acima dos 10 metros das bordas antigas. Embora a densidade de folhas tenha sido uma variável que explicasse significativamente a abundância de espécies pioneiras ao longo da transecção, outras variáveis também são fontes de alteração em ambientes de borda nas florestas ombrófilas como: o vento (Laurance et al. 2002), o tipo de matriz circundante (Nascimento et al. 2006), a idade da borda (Murcia 1995), a orientação geográfica (Kapos 1987), e a disponibilidade de nutrientes (Gómez-Pompa \& Vásquez- 

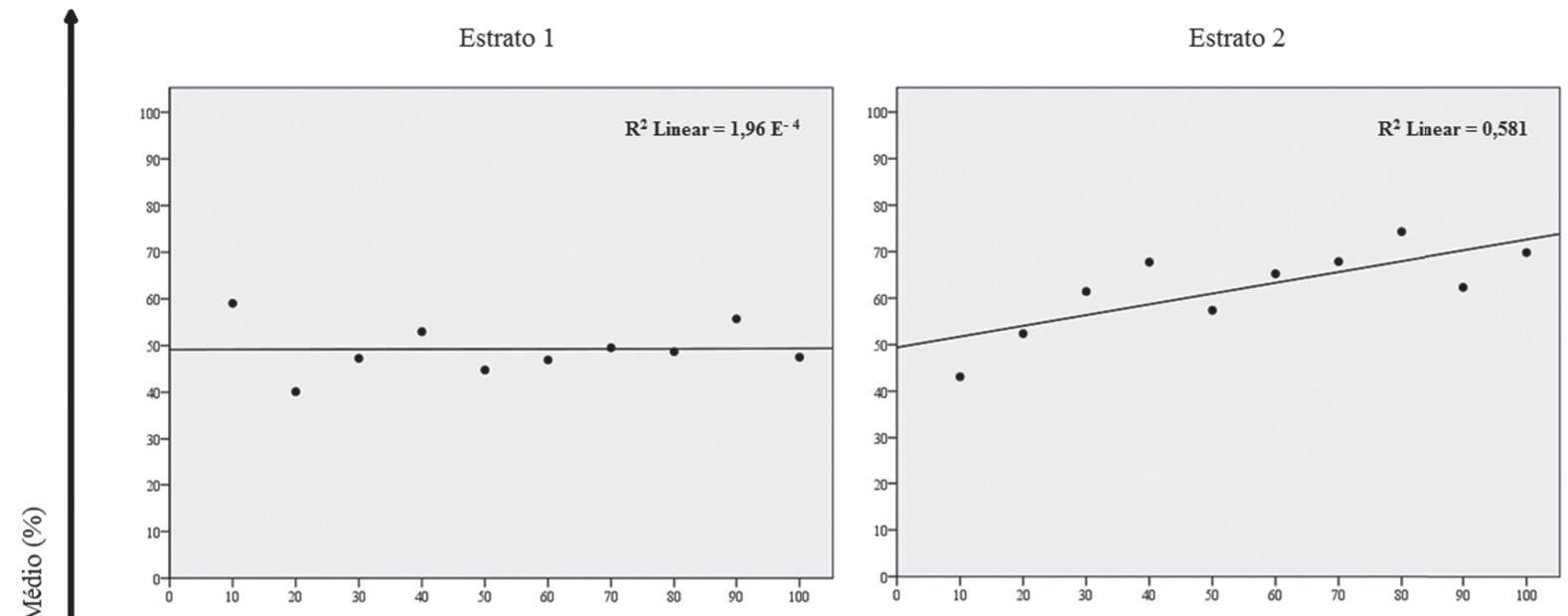

Estrato 3

Estrato 4
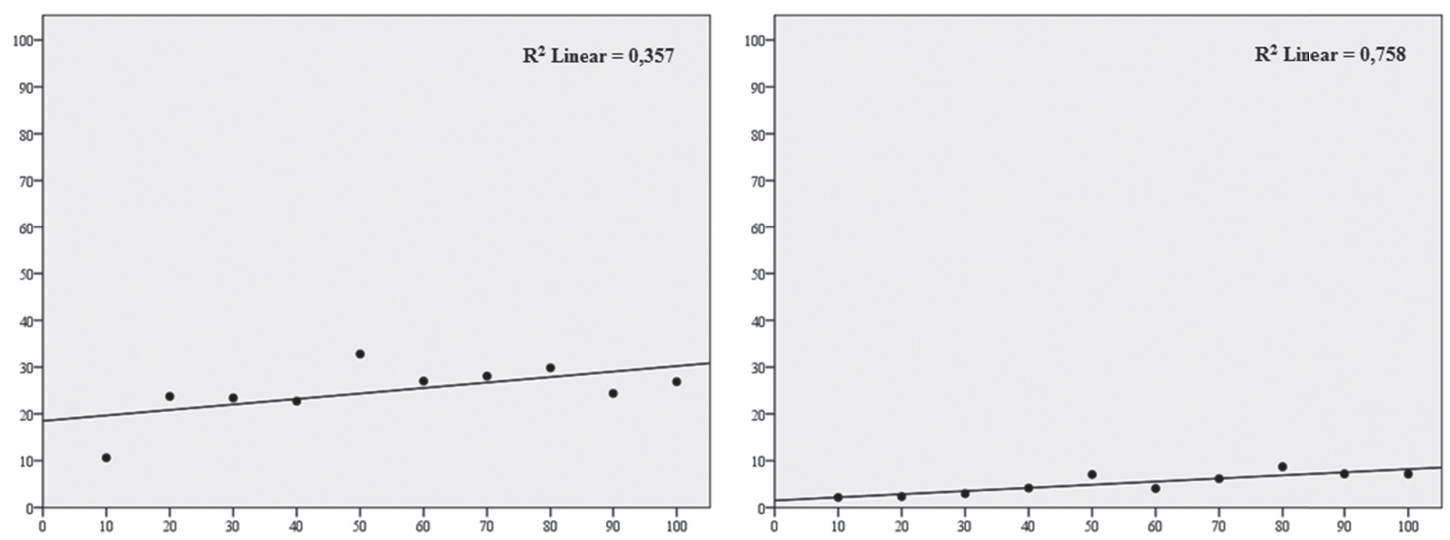

Distância da Borda (m)

Figura 4. Adensamento foliar médio de cada estrato florestal em relação à distância da borda dos fragmentos.

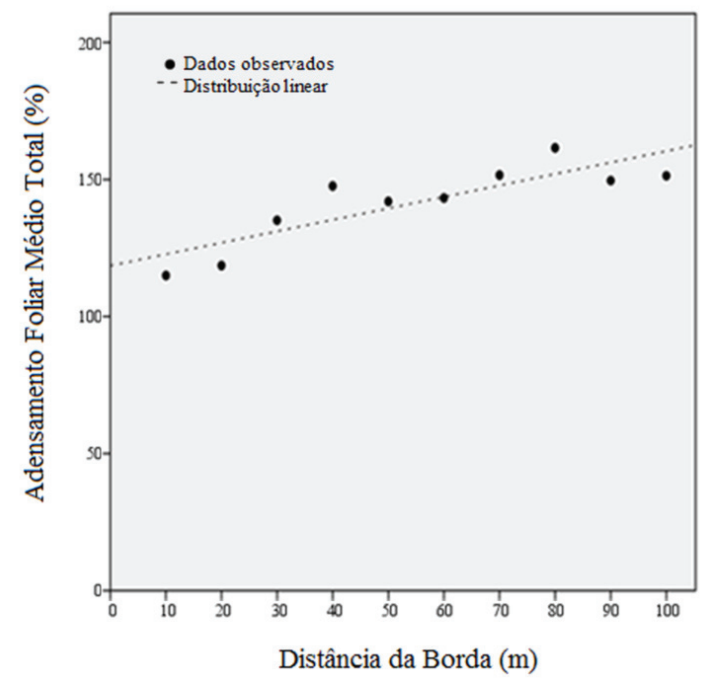

Figura 5. Regressão linear entre o adensamento foliar médio total e a distância da borda dos fragmentos.
-Yanes 1981). Além disso, o tipo de ecossistema é outro fator que influencia os resultados dos efeitos de borda sobre as comunidades vegetais, a exemplo do estudo realizado numa área de Caatinga, onde não foi observado influência da borda sobre o recrutamento ou mortalidade de plantas desse ambiente (Santos \& Santos 2008). Consequentemente, um melhor entendimento acerca da formação de bordas e sua influência sobre comunidades biológicas em fragmentos florestais possibilitará uma melhor aplicação prática dos resultados empíricos obtidos nestes estudos, através do manejo adequado às diferentes realidades das paisagens florestadas tropicais.

\section{Agradecimentos}

Ao Programa de Pós-Graduação em Ecologia e Biomonitoramento da Universidade Federal da Bahia (PPGECO- 
$\mathrm{BIO/UFBA)} \mathrm{pela} \mathrm{possibilidade} \mathrm{da} \mathrm{realização} \mathrm{deste} \mathrm{estudo;}$ aos professores das disciplinas Delineamento Amostral, Métodos de Campo e Bioestatística, Dr. Pedro Rocha, Dr. Francisco Barros e Dr. Eduardo Mariano Neto pelas orientações e revisão preliminar deste artigo; à Prof. MSc Maria Lenise Guedes pelo auxílio taxonômico, à Coordenação de Aperfeiçoamento de Pessoal de Nível Superior (CAPES) pelo financiamento e à equipe da Estação Ecológica de Wenceslau Guimarães - SEMA/BA pelo apoio logístico e acolhimento.

\section{Referências bibliográficas}

Bahia. 2010. Relatório técnico: caracterização da Estação Ecológica Estadual Wenceslau Guimarães. Salvador, Governo do Estado da Bahia, Secretaria do Meio Ambiente.

Bazzaz, F.A. 1979. The physiological ecology of plant succession. Annual Reviews of Ecology, Evolution, and Systematics 10: 351-371.

Brothers, T.S. \& Springarn, A. 1992. Forest fragmentation and alien plant invasion of central Indiana old-growth forests. Conservation Biology 6: 91-100.

Brasil. 1994. Resolução do CONAMA no 5, de 4 de maio de 1994. Define vegetação primária e secundária nos estágios inicial, médio e avançado de regeneração da Mata Atlântica da Bahia. Diário Oficial da União, nº 101, seção 1: 7912-7913.

Dignan, P. \& Bren, L. 2003. Study of the effect of logging on the understorey light environment in riparian buffer strips in a south-east Australian. Forest Ecology and Management 172: 161-172.

Fahrig, L. Effects of habitat fragmentation on biodiversity. 2003. Annual Review of Ecology, Evolution and Systematics 34: 487-515.

Figueiró, A.S. \& Coelho Netto, A.L. 2007. Análise da variabilidade térmica em zonas de bordas florestais com interface urbana no maciço da Tijuca, Rio de Janeiro-RJ. Ciência e Natura 29: 173-186.

Fox, B.J.; Taylor, J.E.; Fox, M. D. \& Williams, C. 1997. Vegetation changes across edges of rainforest remnants. Biological Conservation 82: 1-13.

Franklin, A.B.; Noon, B.R. \& George, T.L. 2002. What is habitat fragmentation? Studies in Avian Biology 25: 20-29.

Gehlhausen, S.M.; Schwartz, M.W. \& Augspurger, C.K. 2000. Vegetation and microclimatic edge effects in two mixed-mesophytic forests fragments. Plant Ecology 147: 21-35.

Gómez-Pompa, A. \& Vázquez-Yanes, C. N. 1981. Successional studies of a rain forest in Mexico. Pp. 247-266. In: West, D.C.; Schugart, H.H \& Botkin, D.B. (Eds.). Forest concepts and application. New York, Springer-Verlag.

Gonzales, M.; Ladet, S.; Deconchat, M.; Cabanettes, A.; Alard, D. \& Balent, G. 2010. Relative contribution of edge and interior zones to patch size effect on species richness: An example for woody plants. Forest Ecology and Management 259: 266-274.

Harper, K.A.; Macdonald, E.; Burton, P.J.; Chen, J.; Brosofske, K.D.; Saunders, S.C.; Euskirchen, E.S.; Roberts, D.; Jaiteh, M.S. \& Esseen, P. 2005 Edge influence on forest structure and composition in fragmented landscapes. Conservation Biology 19(3): 768-782.

Jose, S.; Gillespie, A.R.; George, S.J. \& Kumar, B.M. 1996. Vegetation responses along edge-to-interior gradients in a high altitude tropical forest in peninsular India. Forest Ecology Management 87: 51-62.

Kapos, V., 1989. Effects of isolation on the Waters status of Forest patches in the Brazilian Amazon. Journal of Tropical Ecology 5: 173-185.

Laurance, W.F. 1991. Edge effects in tropical forest fragments: application of a model for the design of nature reserves. Biological Conservation 57: 205-219.
Laurance, W.F. 2000. Do edge effects occur over large spatial scales? Trends in Ecology and Evolution 15: 134-35.

Laurance, W.F.; Lovejoy, T.; Vasconcelos, H.L.; Bruna, E.M.; Didham, R.K.; Stouffer, P.; Gascon, C.; Bierregaard, R.; Laurance, S. \& Sampaio, E. 2002. Ecosystem decay of Amazonian forest fragments, a 22-year investigation. Conservation Biology 16: 605-618.

Laurance, W.F. \& Curran, T.J. 2008. Impacts of wind disturbance on fragmented tropical forests: A review and synthesis. Austral Ecology. 33: 399-408.

Lorenzi, H. 2000. Plantas daninhas do Brasil: terrestres, aquáticas, parasitas e tóxicas. 3 ed. Nova Odessa, Instituto Plantarum.

MacDougall, A. \& Kellman, M., 1992. The understory light regime and patterns of tree seedlings in tropical riparian Forest patches. Journal of Biogeography 19: 667-675.

Matlack, G.R. 1993. Microenvironment variation within and among forest edge sites in the estern united States. Biological Conservation 66: 185-194.

Mourelle, C.; Kellman, M. \& Kwon, L. 2001. Light occlusion at forest edges: an analysis of tree architectural characteristics. Forest Ecology and Management 154: 179-192.

Murcia, C. 1995. Edge effects in fragmented forests: implications for conservation. Trends in Ecology and Evolution 10(2): 58-62.

Nascimento H.E.M.; Andrade A.; Camargo J.L.C.; Laurance W.F.; Laurance S.G. \& Ribeiro J.E.L. 2006. Effects of the surrounding matrix on tree recruitment in Amazonian forest fragments. Conservation Biology 20: 853-60.

Ries, L.; Fletcher Jr., R.J.; Battin, J. \& Sisk, T.D. 2004. Ecological responses to habitat edges: mechanisms, models, and variability explained. Annual Review of Ecology, Evolution, and Systematics 35: 491-522.

Sanqueta, C.R. 1995. Análise da estrutura vertical de florestas através do diagrama h-M. Ciência Florestal 5(1): 55-68.

Santos, A.M.M., Santos, B.A. 2008. Are the vegetation structure and composition of the shrubby Caatinga free from edge influence? Acta Botanica Brasilica 22(4): 1077-1084.

Souza, V.C. \& Lorenzi, H. 2005. Botânica sistemática: guia ilustrado para identificação das famílias de Angiospermas da flora brasileira, baseado em APG II. Nova Odessa, Instituto Plantarum.

SPSS. 2010. SPSS Statistics for Windows v. 17. Chicago, Illinois, EUA.

Sheskin, D. 2000. Handbook of parametric and nonparametric statistical procedures. Second edition. New York, Chapman \& Hall/CRC.

Sprent, P. 2001. Applied nonparametric statistical methods. Third edition. New York, Chapman \& Hall/CRC.

Tabarelli, M.; Mantovani, W. \& Peres, C.A. 1999. Effects of habitat fragmentation on plant guild structure in the montane atlantic forest of southeastern Brazil. Biological Conservation 91: 119-127.

Tabarelli, M.; da Silva, J.M.C. \& Gascon, C. 2004. Forest fragmentation, synergisms and the impoverishment of neotropical forests. Biodiversity and Conservation 13: 1419-1425.

Tabarelli, M.; Aguiar, A.V.; Grillo, A.S. \& Santos, A.M.M. 2006. Fragmentação e perda de habitats na Mata Atlântica ao norte do Rio São Francisco. In: Fragmentos de mata atlântica do nordeste biodiversidade, conservação e suas bromélias. Siqueira Filho, J.A. Leme, E.M.C. Rio de Janeiro, Andrea Jakobsson Estúdio.

Valladares, F.; Wright, S.J.; Lasso, E.; Kitajima, K. \& Pearcy, R.W. 2000. Plastic phenotypic responses to light of 16 congeneric shrubs from Panamanian rainforest. Ecology 81: 1925-1936.

Watling, J.I. \& Orrock, J.L. 2010. Measuring edge contrast using biotic criteria helps define edge effects on the density of an invasive plant. Landscape Ecology 25: 69-78.

Williams-Linera, G.; Dominguez-Gastelu, V. \& Garcia-Zurita, M.E. 1998. Microenvironment and floristics of different edges in a fragmented tropical rainforest. Conservation Biology 1: 33-41.

Versão eletrônica do artigo em www.scielo.br/abb e http://www.botanica.org.br/acta/ojs 